\title{
Student Trajectories for Enrollment Forecasting, Management, and Planning
}

\author{
R. Alan Bowman
}

\section{About the Author}

R. Alan Bowman is professor of operations and information systems at the Capital Region Campus of Clarkson University in Schenectady, New York.

\begin{abstract}
The School of Management (SOM) at Union Graduate College (UGC) used student trajectories to forecast both individual course and total enrollments within and across various student categories. (The term "trajectory" refers to the enrollment pattern of the average student in a particular category.) The trajectories and the resultant projections formed an integral part of diagnostic tools for enrollment management decision-making, short- and long-term course section planning, and faculty capacity analyses in a variety of scenarios to inform program development, program promotion, and faculty hiring, and to make sure they were consistent with each other. This article describes the concepts behind trajectories, various types of trajectories and their respective uses, details for constructing trajectories, and how trajectories can be incorporated in reports and tools for maximum utility.
\end{abstract}

Keywords: enrollment forecasting, enrollment management, course section planning, faculty capacity planning

The AIR Professional File, Spring 2021

Article 153 https://doi.org/10.34315/apf1532021

Copyright (c) 2021, Association for Institutional Research 


\section{INTRODUCTION}

Enrollment forecasting and planning in higher education has received considerable attention both in isolation and as an integrated component of institutional planning; for example, see the early and comprehensive treatment by Hopkins and Massy (1981). A wide variety of approaches to enrollment forecasting and planning have been described over the years. Most methodological descriptions have been accompanied by specific applications with various differentiating factors both in terms of the setting and of the desired practical uses. Although each institution is unique, the hope of each article is that there is enough commonality that each approach will be useful to multiple institutions albeit with appropriate modifications as needed. It is with this hope that I describe an approach that was developed in the School of Management (SOM) at Union Graduate College (UGC) prior to its merger with Clarkson University in 2016. This approach has some similarities both in structure and in goals with prior approaches, as I will describe when I review relevant literature and describe UGC's approach in detail, but is fundamentally different. At this point, I summarize the most important characteristics of UGC's setting and planning needs.

The SOM offered several master's degrees, including both a master of business administration (MBA) and a health-care MBA; it also offered certificates. The number of courses, as well as the specific courses, required varied between degrees and between students within a degree due to course waivers and/ or courses transferred in. There were both part-time and full-time students. Student objectives in terms of how quickly they wanted to complete their degrees also varied greatly not only between degrees and between full-time and part-time students, but also between full-time students within the same degree and between part-time students within the same degree. The academic calendar included three trimesters during the regular year plus a summer term. Admissions were on a rolling basis so that students could begin their studies in any term.

All of the classes were in the evenings or online so they could be attended by full-time and part-time students. Most of the courses were part of multiple degree or certificate programs. The total volume of enrollments from all programs allowed the SOM to offer 80 to 90 course sections per year with average enrollments of 16-18 per section.

The desired capabilities of a model were extensive and varied. Students were charged tuition on a per course basis so, although projecting the number of students was important, it was more important to project the number of course enrollments. It was also desired to project the number of enrollments in specific courses, but doing so was for the purpose of deciding the number of sections of each course that would need to be offered rather than predicting any specific course section's enrollment. Diagnostic capabilities for forecast errors were needed to help focus remedial actions such as retention strategies. The relationship between student mix (both by program and by full time vs. part time) and course enrollments (both total and by specific course) needed to be a part of the model to allow marketing and other program promotions to focus on student categories that were compatible with resource capabilities in both the short and long terms. This meant that the model needed to at least provide the necessary inputs for successful planning of faculty size and configuration.

The SOM at UGC devoted very limited resources to institutional research and planning. There was a head of enrollment planning for the entirety of 
UGC and a recruiter/enrollment manager devoted to the SOM. Those two worked together to track indicators of new student recruitment as well as student retention. I developed the models described here in part while working as a full-time professor of operations management/operations research at the SOM and more fully while working as a half-time professor and half-time associate dean of the SOM. The data available were limited to what the head of enrollment planning and the recruiter/enrollment manager could provide, plus historical student records from the student information system. In summary, the model needed to satisfy a variety of needs but had to be relatively simple, easy to use, and easy to update and maintain.

\section{LITERATURE}

Chen (2008) provides a thorough review of the main approaches to enrollment forecasting that have been used in the past: subjective judgment, ratio method, cohort survival, Markov chains, neural networks, simulation, time series, fuzzy time series, and regression. His paper provides examples of and compares time series and regression and ends with very similar models to predict the number of students enrolled. Another good example of time series analysis is provided by Lavilles and Arcilla (2012). Those authors also predict the number of students enrolled; that indicator seems to be the main focus of the literature with the assumption that the number of enrollees is more important than the total number of course enrollments or that the latter follows naturally from the former. Neither of these assumptions was true at UGC. It should be noted, however, that the nature of both time series and regression is such that they could be used equally well for total course enrollments. Neither approach, however, lends itself very well to the diagnostic and prescriptive capabilities desired by UGC.

Markov chain models, on the other hand, more naturally provide the capability for both diagnosis and prescription via the transition probabilities that are at the heart of their structure. In a sense, they are the extension of ratio and cohort models to allow the modeling of more-complex, but common, behaviors, such as reentry. Although the typical transitions refer to students moving from first year to second year, for example, and eventually to either program completion or dropping out, the state definitions can be extended to help meet needs specific to particular applications. Gandy et al. (2019) add cumulative credit hour ranges and Rahim et al. (2013) add age group ranges to their state definitions to gain additional insights as well as predictive power. Nicholls (2007) specifically focuses on the use of the Markov chain model for improving the program completion results for master's and PhD students, and also identifies the usefulness of the models for longer-term analyses. The model developed at UGC has similarities with Markov chain models but is structured around course enrollments, which are related to student enrollments but have additional complexities that need to be taken into account. Shapiro and Bray (2011) discuss what they call a de-cohortized approach developed specifically for part-time programs at Northwestern University that uses transition matrices and is primarily based on the length of time an individual has been in the program. As I do in this article, those authors emphasize the prescriptive capabilities of their model beyond its forecasting use.

There are also a number of extremely useful reports and slide presentations from conferences that describe the settings as well as the approaches 
taken at various colleges and universities. Several also provide good reviews of the field before describing their own approach. Reiss (2012) provides a particularly good review before describing the specific approach at the University of Central Florida. Examples of other good reports and presentations include Redlinger et al. (2013, presentation based on University of Texas, Dallas), Link and Whitford (2018, presentation based on University of Buffalo), Rylee and Trusheim (2004, presentation based on University of Delaware), and the Maryland Higher Education Commission (2016, report on Maryland public colleges and universities).

UGC also needed to forecast enrollments in specific courses; this topic has received attention in the literature as well. These forecasting efforts have typically been separate from overall enrollment projections and have been shorter term in nature. These efforts take into account characteristics of specific students in the programs and, in some cases, consider information about the course offerings as well. For example, Balachandran and Gerwin (1973) offered three approaches based on including just the first, the first two, or all three of the following variables to divide the students into categories: (1) if the student has taken the course, (2) if the student has taken the prerequisites for the course, and (3) if the course is required in the student's major. Kraft and Jarvis (2005) included GPA in prerequisite courses and other groupings relevant to specific courses. Ognjanovic et al. (2016) included many demographics as well as course-specific information such as the scheduled time the course was held, who the professor was, and even teaching evaluation scores. UGC's needs in this area were longer term in nature, and were used for section planning rather than for room or term schedule planning.
The SOM at UGC needed features of all these models and wanted the model(s) to be as simple as possible and to take into account the specific characteristics of the setting that I previously described. I will discuss ways that the model developed at UGC could be extended or complemented by other models, but for now I turn to describing the UGC model itself and how it was developed and used.

\section{STUDENT TRAJECTORIES}

At the heart of UGC's enrollment forecasting approach is what I refer to as student trajectories. The main objective of this article is to introduce trajectories to the literature, including the wide variety of tools trajectories enable. The trajectory for each student category can then be applied to the number of students in each category, both actual matriculated students and forecasted incoming students, to generate enrollment projections. This concept requires more explanation but it is important to note up front that the data requirements for each student are their transcript, student category, and date of matriculation.

The building block of all the student trajectories, from which all enrollment projections are generated via various multiplications and summations, is a matrix $C_{i j}(r y, t)$. There are seven rows with ry referring to the year relative to student i's year of matriculation running from $r y=1$ (the actual year of matriculation) up through $r y=7$ (the 7th year of being a matriculated student). There are four columns with $t$ referring to the term $(t=1,2,3$, and 4 for Winter, Spring, Summer, and Fall, respectively). Each element of the matrix for student $i$ and course $j$ is given by 


$$
\begin{aligned}
& C_{i j}(r y, t)=1 \text { if student } i \text { took course } j \text { in term } t \text { of their relative year } r y \text {, or } \\
& =0 \text { if they did not, or } \\
& =\text { (missing value) if term } t \text { of their relative year ry has not yet occurred in the database. }
\end{aligned}
$$

Essentially, this matrix is O's except for a 1 placed in the row (relative year) and column (term) corresponding to when student $i$ took course $j$, although it could be all o's if student $i$ has not taken course $j$. The matrix could also have more than a single 1 if student $i$ took course $j$ more than once. It is crucial to the trajectory calculations to recognize that $r y$ is an index relative to each student's year of matriculation and that the vector element is a missing value rather than 0 if the term has not yet occurred.

These $C_{i j}$ matrices are then summed across all courses $j$ for each student $i$ to yield the total number of courses student $i$ took in term $t$ of their relative year $r y$ (again, a missing value if that term has not yet occurred),

\section{$E_{i}(r y, t)=\Sigma_{\mathrm{j}} C_{\mathrm{ij}}(r y, t)$,}

so that student i's total course enrollment $E_{i}$ matrix also has seven rows and four columns. The $C_{i j}$ and $E_{i}$ matrices for each student are then averaged across all students in a student category $x$ to derive the student course trajectory matrix $\left(\operatorname{SCT}_{x}(r y, t)\right)$ and the student enrollment trajectory matrix $\left(\operatorname{SET}_{x}(r y, t)\right)$, respectively, for that category $x$. Note that older student records in the database will have full matrices (i.e., all elements will be 0 or 1), whereas more-recent student records will have only partial matrices (i.e., missing values for some elements since those terms will not yet have occurred). The missing values should be omitted from the averages. The default for most averaging functions provided in software packages is to average in O's but not missing values; it is crucial to make sure this convention is followed.

Although the student course trajectories are very helpful and I will describe how UGC used them later in this article, it is the student enrollment trajectories that are likely of primary interest to most readers so I start with them. The $\operatorname{SET}_{x}(r y, t)$ trajectory coefficients should be somewhat intuitive although their magnitudes may be surprising in the sense that one tends to think of enrollments based on a student who completes the entire program with no course waivers or transfers and who takes a steady number of courses each term until done. To get a feel for the student trajectory coefficients, I display them for the full-time MBA category $(x=9)$ in Table 1. 
Table 1: Trajectory Coefficients for Full-Time MBA Students

\begin{tabular}{|c|c|c|c|}
\hline Coefficient & Year & Term & Average Number of Courses Per Student \\
\hline $\operatorname{SET}_{9}(1,1)$ & Matriculation Year & Winter & 0.198 \\
\hline $\operatorname{SET}_{9}(1,2)$ & Matriculation Year & Spring & 0.335 \\
\hline $\operatorname{SET}_{9}(1,3)$ & Matriculation Year & Summer & 0.457 \\
\hline $\operatorname{SET}_{9}(1,4)$ & Matriculation Year & Fall & 2.551 \\
\hline $\operatorname{SET}_{9}(2,1)$ & Matriculation Year + 1 & Winter & 2.566 \\
\hline $\operatorname{SET}_{9}(2,2)$ & Matriculation Year + 1 & Spring & 2.411 \\
\hline $\operatorname{SET}_{9}(2,3)$ & Matriculation Year + 1 & Summer & 0.916 \\
\hline $\operatorname{SET}_{9}(2,4)$ & Matriculation Year + 1 & Fall & 1.832 \\
\hline $\operatorname{SET}_{9}(3,1)$ & Matriculation Year + 2 & Winter & 1.244 \\
\hline$S E T_{9}(3,2)$ & Matriculation Year + 2 & Spring & 0.900 \\
\hline $\operatorname{SET}_{9}(3,3)$ & Matriculation Year +2 & Summer & 0.225 \\
\hline $\operatorname{SET}_{9}(3,4)$ & Matriculation Year +2 & Fall & 0.334 \\
\hline $\mathrm{SET}_{9}(4,1)$ & Matriculation Year + 3 & Winter & 0.214 \\
\hline $\operatorname{SET}_{9}(4,2)$ & Matriculation Year + 3 & Spring & 0.089 \\
\hline $\operatorname{SET}_{9}(4,3)$ & Matriculation Year + 3 & Summer & 0.009 \\
\hline $\operatorname{SET}_{9}(4,4)$ & Matriculation Year + 3 & Fall & 0.027 \\
\hline $\mathrm{SET}_{9}(5,1)$ & Matriculation Year +4 & Winter & 0.034 \\
\hline $\mathrm{SET}_{9}(5,2)$ & Matriculation Year + 4 & Spring & 0.023 \\
\hline $\mathrm{SET}_{9}(5,3)$ & Matriculation Year +4 & Summer & 0.011 \\
\hline
\end{tabular}

As noted, the coefficients were actually computed for 7 years for all categories but are insignificant for this category beyond what is shown. Some reflection on the trajectory coefficients should make it clear that they take into account not only student retention (similar to ratio/cohort/Markov chain models, including withdrawal and reentry) but also the speed with which students take courses and how many courses they take in total. The latter indicator depended not only on which program students were in but also any course waivers and transfers they were granted plus the occasional extra courses they took beyond degree requirements. Note that the total of the right column is 14.178 whereas the program required 17 
courses. The difference reflects all of the previously described factors in their proper proportions. This is why the trajectory coefficients can be quite different from what one might at first expect. A key aspect of the trajectories is that they accomplish all of this with fairly simple tabulations and summations. Building separate models to adequately address each factor would be quite cumbersome.

UGC's most important objective was to forecast total course enrollments across all students and categories. To convert the trajectory coefficients into total course enrollment projections is a matter of fairly intuitive multiplications of the coefficients by new matriculant numbers across student categories and years. Since UGC computed the trajectories for 7 years, we multiplied them by 7 years of new matriculants. To project the course enrollments for any category $x$ for year $y, U G C$ used the matriculant vector $M_{x y^{\prime}}$ where $M_{x y}(1)$ is the number of matriculants in category $x$ in year $y, M_{x y}(2)$ is the number of matriculants in category $x$ in year $y-1$, $\ldots$, and $M_{x y}(7)$ is the number of matriculants in category $x$ in year $y-6$. The elements of this vector were known values for years that had already occurred but forecasted values for those that had not. Again, letting $\mathrm{t}=1,2$, 3, or 4 for the Winter, Spring, Summer, and Fall, respectively, UGC computed the total projected enrollments from all students in category $x$ in term $t$ of year $y$ as

$$
T E_{x y}(t)=\Sigma_{k=1}^{7} \mathrm{M}_{x y}(k) S E T_{x}(k, t) .
$$

The total enrollments projection from students in category $x$ for the entire year $y$ was then obtained by summing across the four terms. Summing the individual term projections across all categories yielded the total enrollment projection for each term and summing the total enrollment projections for each term yielded the grand total enrollment projection for year $y$.

To exemplify the calculations, suppose one is trying to project the enrollments from MBA management fulltime students in year 2022; the projected or known new matriculants in this category are given in Table 2.

\section{Table 2: New Matriculants for Full-Time MBA Students}

\begin{tabular}{ccc}
\hline Coefficient & Year & New Matriculants \\
\hline$M_{9,2022}(1)$ & 2022 & 45 (projected) \\
$M_{9,2022}(2)$ & 2021 & 47 (projected) \\
$M_{9,2022}(3)$ & 2020 & 26 \\
$M_{9,2022}(4)$ & 2019 & 32 \\
$M_{9,2022}(5)$ & 2018 & 48 \\
$M_{9,2022}(6)$ & 2017 & 51 \\
$M_{9,2022}(7)$ & 2016 & 35
\end{tabular}


The projected enrollments from this student category in the Fall term of year 2022 would be

$$
\begin{gathered}
\operatorname{TE}_{9,2022}(4)=M_{9,2022}(1) \operatorname{SET}_{9}(1,4)+M_{9,2022}(2) \operatorname{SET}_{9}(2,4)-M_{9,2022}(3) \operatorname{SET}_{9}(3,4) \\
+M_{9,2022}(4) \operatorname{SET}_{9}(4,4)+M_{9,2022}(5) \operatorname{SET}_{9}(5,4)+M_{9,2022}(6) \operatorname{SET}_{9}(6,4)+M_{9,2022}(7) \operatorname{SET}_{9}(7,4) \\
=45 * 2.551+47 * 1.832+26 * .334+32 * .027+48 * 0+51 * 0+35 * 0=115+86+8+1=210 .
\end{gathered}
$$

Similar computations would be done for all student categories and for each term. The interested reader can verify that the projected enrollments for the other three terms in this year would be

$$
T E_{9,2022}(1)=170, T E_{9,2022}(2)=156 \text {, and } T E_{9,2022}(3)=70 \text {. }
$$

Thus, the total projected enrollments from this student category in the year 2022 would be 606. Similar calculations would be done for each student category and the total projected enrollments summed across all categories to yield the grand total enrollment projection.

The student enrollment trajectories are easily modified to reflect active student trajectories by first substituting 1 (indicating the student was active) for any positive number of courses taken into each student's total course taken matrix. In other words, let

\section{$A_{i}(r y, t)=1$ if $E_{i}(r y, t)>1$, or $=E_{i}(r y, t)$, otherwise,}

so that $A_{i}(r y, t)=1$ if student $i$ was active in term $t$ of their relative year $r y, 0$ if not, and a missing value if their term $t$ had not yet occurred.

These active student vectors are then averaged across all students in a category $x$ (again omitting missing values) to yield the active student trajectory $A S T_{x^{\prime}}$ where $A S T_{x}(r y, t)$ is the average proportion of students in category $x$ active in term $t$ of their relative year $r y$. Then one simply repeats the calculations I have just described using the active student $A S T_{x}$ matrix in place of the student enrollment trajectory $S E T_{x}$ matrix to get the projected number of active students in each category each term in the year $y$ being projected.

Student categories should be selected to divide students into groups with similar trajectories in terms of total courses, specific courses, and time in program. Obvious ways to do this are based on the degree sought and part-time versus full-time status. Other categorizations may be useful for students who have matriculated (such as term started or number of courses already taken) but would likely not be feasible for use with projected matriculants. It should also be kept in mind that the number of students in a category needs to be large enough for the trajectory coefficients to be reliable. I will say a bit more on this later. UGC used the matriculant categories in Table 3. 
Table 3: Matriculant Categories
1. Certificates
2. Juris Doctor/MBA Management
3. Leadership in Medicine/MBA Full Time
4. Leadership in Medicine/MBA Part Time
5. 5 Year Undergraduate/MBA
6. MBA Health Care Full Time
7. MBA Health Care Part Time
8. 5 Year Undergraduate/Health-Care MBA
9. MBA Management Full Time
10. MBA Management Part Time
11. 5 Year Undergraduate/MBA Part Time
12. Pharmacy Doctor/MS Health Care
13. Pharmacy Doctor/MBA Health Care
14. Accounting MBA
15. MS Health-Care Data Analytics Full Time
16. MS Health-Care Data Analytics Part Time

Note: MS is master of science.

The master of science (MS) health-care data analytics program was a new program introduced after the enrollment projection and planning approach had been implemented so that there were no data directly from the program initially to use to form a trajectory for the final two categories. UGC found, however, that using knowledge of the program structure and trajectories for other student categories as a guide, and developing a trajectory for initial use for Categories 15 and 16 was more intuitive and accurate than relying on a purely subjective estimate based on hypothesized average student behavior or, even worse, the trajectory of a hypothesized typical student in that category.

\section{NEW MATRICULANT PROJECTIONS AND THE FUNNEL MODEL}

The calculations described in the preceding section are the same whether the number of matriculants in a year is a known number (an already completed year) or a forecast (the current or future year). If it is a forecast, it might be for the purpose of making enrollment forecasts that are as accurate as possible. It might also be used to set goals or to be part of a sensitivity analysis for strategic planning. I will discuss the latter uses more below, but note now that the trajectories are well suited for all these purposes.

Although the main objective of this article is to introduce the use of student trajectories that can be combined with matriculant projections no matter how the latter are obtained, I will briefly describe UGC's approach to new matriculant forecasting. UGC used a fairly standard approach that combined ratios with subjective judgment in a funnel model. The funnel model tracked students from inquiries all the way to actual matriculation and measured the percentage that advanced to each step (which declines as the steps advance, hence the term "funnel"). The steps that UGC tracked were as follows:

1| Student inquires about the program.

2) Student applies to the program. 
3| Student receives admission acceptance letter.

4| Student submits deposit.

5| Student enrolls in first course as a matriculated student.

The funnel model was tracked separately for fulltime and part-time students and for foreign and US students since the percentage of students that moved from step to step varied significantly across these categories. The funnel percentages for US students just prior to the merger were as shown in Table 4.

\section{Table 4: Percent of Inquiries Advancing}

\begin{tabular}{cc}
\hline Step & \% of Inquiries \\
\hline Applied & 31.9 \\
Admitted & 25.0 \\
Submitted deposit & 21.1 \\
Matriculated & 20.6 \\
\hline
\end{tabular}

Not only were numbers of students that advanced through each step recorded for entire years, but the counts were also recorded on a biweekly basis throughout each year. This database enabled projections to be made at any time from several different bases. For example, suppose one wanted to project the number of full-time US matriculated students for 2020 based on the number of applications received for 2020 as of the end of March 2020, and suppose this number was 50 . This base would first be used to project the total number of
2020 applications as follows:

Projected total number of US full-time applications for $2020=$

(Number of US full-time applications at the end of March, 2020) /

(Historical proportion of total annual US full-time applications received by the end of March) =

$$
50 / .80=63 .
$$

The projected number of matriculated students would then be made as follows:

Projected matriculated students for $2020=$ (Projected total number of applications for 2020) * (Historical proportion of applications that result in matriculated students) $=$

$$
63 * 20.6 / 31.9=41 .
$$

The counts at any point in time for any of the five steps could be used in a similar manner to project the number of matriculated students. If the percent yields (i.e., the percent advancing from one step to the next) were consistent with historical data, the projections from each base would be consistent as well. Of course, another objective of the funnel model was to actually increase the yield percentages through improvements in the process of managing the students from inquiry to matriculation. For the purposes of projection accuracy, the possibility of funnel improvements favored using later steps rather than earlier steps as bases.

The head of enrollment planning and the recruiter/ enrollment manager worked together to subjectively 
modify the numbers (either the input percentages or the output projections directly) so that they were always some combination of a historical ratio and a subjective model. Especially for longer-term enrollment projections, either very early in the year being projected or for future years as part of a long-term outlook, the matriculant projections were used in sensitivity analyses that were useful for setting student category-mix adjusted-growth goals consistent with both market forecasts and resource planning.

\section{DIAGNOSTICS AND CONTROL MEASURES}

One of the significant advantages of the trajectories compared to many other projection approaches is the ability to use the trajectory model for diagnostic and prescriptive rather than just predictive purposes. Once the enrollment numbers were known for a term, UGC produced a report for each category comparing the actual and predicted. An example report (once again for the full-time MBA management student category) is shown in Table 5.

Table 5: Example Diagnostic Report for Full-Time MBA Students Fall Term

\begin{tabular}{|c|c|c|c|c|}
\hline & \multirow{2}{*}{\multicolumn{4}{|c|}{ Year of Matriculation }} \\
\hline & & & & \\
\hline & $20 x x$ & $20 x x-1$ & $20 x x-2$ & $20 x x-3$ \\
\hline Number of Students & 45 & 47 & 26 & 32 \\
\hline \multicolumn{5}{|l|}{ Model: } \\
\hline Percent of Students Active & 86 & 73 & 20 & 3 \\
\hline Enrollments per Active Student & 2.96 & 2.51 & 1.67 & 1 \\
\hline Enrollments per Student & 2.55 & 1.83 & 0.33 & 0.03 \\
\hline Number of Enrollments & 115 & 86 & 9 & 1 \\
\hline \multicolumn{5}{|l|}{ Actual: } \\
\hline Percent of Students Active & 92 & 58 & 16 & 0 \\
\hline Enrollments per Active Student & 3.09 & 2.46 & 1.50 & 0 \\
\hline Enrollments per Student & 2.84 & 1.44 & 0.24 & 0 \\
\hline Number of Enrollments & 128 & 68 & 6 & 0 \\
\hline Enrollments (Actual-Model) & 13 & -18 & -3 & -1 \\
\hline
\end{tabular}


In this case, the report showed that full-time students were slightly increasing the speed at which they took courses, resulting in more enrollments in the first year and fewer in subsequent years. This behavior was viewed as a positive and the main discussion centered on whether this was going to be an ongoing pattern such that the trajectories should be modified. There was some concern that the percent of active students that matriculated the previous year was down. Investigations revealed this downturn was mostly due to a variety of individual circumstances and did not warrant system changes.

For the same term, the diagnostic report for parttime MBA students showed that the enrollments per active student had dropped, resulting in a total of 14 fewer enrollments than predicted. Students were contacted and the main factor in the slowdown was determined to be employer reimbursement policies becoming more restrictive. Program administrators worried that this would eventually lead to losses in retention and discussed possible remedies such as loans or increased scholarship opportunities.

\section{COURSE PLANNING}

The literature on projecting enrollments in specific courses is essentially separate from that of projecting total enrollments but the trajectories allowed UGC to accomplish both in essentially the same manner. I have described in detail the calculations for projecting total enrollments; to project the enrollments in an individual course was simply a matter of doing the same calculations using the student course trajectory coefficients $\left(S C T_{x}(r y, t)\right)$ in place of the student enrollment trajectory coefficients $\left(\operatorname{SET}_{x}(r y, t)\right)$. The resulting totals for a particular term for a particular course very much depended on whether that course was offered in that term, perhaps with more than one section; the results had to be interpreted with that in mind. The results for an entire academic year were more meaningful, which is how UGC primarily used them to make decisions about how many sections of each course to offer. The specific course trajectories reflected all the factors for the enrollment trajectories but also naturally captured the waiver/ transfer likelihood for the specific course as well as the probability a student would leave before taking the course; that likelihood was higher for courses taken late in a program.

UGC's approach for determining the number of course sections of each course started with selecting a capacity for each course. This was 30 for most courses, although there was some variety based on the nature of each course. UGC used 30 for all electives (courses not required in any student category) and lumped the enrollments in these electives together to determine the total number required; specific elective choices were based on knowledge of both student preferences (demand) and faculty expertise (supply). The maximum average enrollments per section for each course (maximum average) was then set at a consistent percentage of the capacity. The precise calculation was then done by dividing the total projected enrollments for each course by the maximum average and rounding up. For example, suppose the percent of capacity was 81 so that the maximum average was $81 \% \times 30=24.8$ for the MBA500 course, and suppose that the enrollment projection for this course for the entire year was 93. This means that UGC desired to have the average number of enrollments per section of MBA500 be 24.8 or less so the number of sections of MBA500 would then be $(93$ / 24.8) rounded up, which is 4 . 
It is the average number of enrollments per section (i.e., the average class size), however, that is typically of more interest and that is more easily understood than either the maximum average or the percent of capacity. Average class size is often decided on as a matter of program policy that involves many factors and may change over time. To help in making this decision, UGC produced a trade-off table (see example in Table 6) that showed the percent of capacity the maximum average would have to be to achieve a range of average class sizes and the corresponding number of sections. The full table would show the results for every course and the total at the bottom. This table was created by starting the maximum average at full capacity (100\%) and reducing the percent in 0.1 increments. For each increment, the resultant total number of sections across all courses was divided into the total enrollments to get the average class size that would result from that percent. The percent that corresponded to various average class sizes was then placed in a table such as Table 6. The table helped UGC make a final decision by selecting a desired column. For example, suppose UGC's desired average was 18 students per section. The maximum average for MBA500 would be $71.7 \% \times 30=21.5$, and there would be $93 / 21.5=4.3$ rounded up to five sections of MBA500 with an average enrollment of $93 / 5=18.6$.

\section{Table 6: Trade-Off Table for Number of Sections}

\begin{tabular}{|c|c|c|c|c|c|c|}
\hline & \multicolumn{6}{|c|}{ Average Class Size } \\
\hline & 15 & 16 & 17 & 18 & 19 & 20 \\
\hline Maximum Average \% of Capacity & 58.3 & 62.0 & 67.0 & 71.7 & 76.0 & 81.0 \\
\hline Sections of MBA500 & 6 & 6 & 5 & 5 & 5 & 4 \\
\hline
\end{tabular}

\section{FACULTY PLANNING}

It is intuitive that determining the number of sections of each course is very useful for conducting faculty planning. In UGC's case, the number of sections was linked to faculty planning via the Association to Advance Collegiate Schools of Business (AACSB) requirements for faculty coverage since the SOM of UGC was AACSB accredited. AACSB mandated that certain percentages be covered by participating faculty (i.e., faculty involved with the program for more than teaching, who were essentially nonadjunct faculty) both by disciplinary areas and in total. Table 7 shows the computations done by UGC for the upcoming year prior to the merger. The adequacy of coverage both by area and in total was easily seen and helped inform hiring decisions. 
Table 7: Faculty Coverage

\begin{tabular}{|c|c|c|c|}
\hline \multirow{2}{*}{ Area } & \multirow{2}{*}{ Projected Sections } & \multicolumn{2}{|c|}{ Participating Faculty Coverage } \\
\hline & & Sections & Percent \\
\hline \multicolumn{4}{|c|}{ Finance/Accounting/Economics } \\
\hline Required & 13 & 9 & 69 \\
\hline Elective & 9 & 7 & 78 \\
\hline Total & 22 & 16 & 73 \\
\hline \multicolumn{4}{|c|}{ Marketing/Operations/Management Science } \\
\hline Required & 14 & 11 & 79 \\
\hline Elective & 10 & 7 & 70 \\
\hline Total & 24 & 18 & 75 \\
\hline \multicolumn{4}{|c|}{ Management/Human Resources } \\
\hline Required & 13 & 11 & 85 \\
\hline Elective & 15 & 12 & 80 \\
\hline Total & 28 & 23 & 82 \\
\hline \multicolumn{4}{|l|}{ Health-Care Management } \\
\hline Required & 7 & 6 & 86 \\
\hline Elective & 11 & 6 & 55 \\
\hline Total & 18 & 12 & 67 \\
\hline \multicolumn{4}{|l|}{ Totals } \\
\hline Required & 47 & 37 & 79 \\
\hline Elective & 45 & 32 & 71 \\
\hline Total & 92 & 69 & 75 \\
\hline
\end{tabular}

\section{INTERMEDIATE AND LONG- TERM PLANNING}

I mentioned earlier that the matriculant projections were sometimes intended to be the most accurate estimates available and were sometimes viewed more as goal numbers. For intermediate and longterm planning, the process that was developed using the trajectory model to convert matriculant numbers into course section and faculty planning reports as shown in Tables 6 and 7 was ideal. The operational ramifications of various growth strategies (for some categories) and contraction strategies (for others) could be easily seen. New programs (categories) could be included in this type of analysis by developing hypothetical trajectories as UGC did 
when the MS health-care data analytics program was introduced. The result was that sensitivity analysis on faculty resource requirements was easy to conduct and easy to properly consider in planning exercises. Note that the trajectories varied by category both in terms of specific courses taken and in the timing patterns, so that the planning reports of Tables 6 and 7 needed to be done for multiple years to reflect the transient as well as steady state effects. The trajectories enabled these planning exercises to be done in a very natural manner.

\section{ACCURACY AND USEFULNESS}

There were two main insights from UGC's use of trajectories (and the funnel model) in terms of accuracy. The first was that projecting new matriculants (e.g., using the funnel model) was by far the most difficult part and prone to error. The trajectories were more accurate, which meant that enrollment projections from continuing students were more accurate than projections from new students. In the first 2 years of implementation, the new matriculant projections prior to the start of the academic year were accurate enough that the grand total enrollment projections across all student categories for the entire year were within $1 \%$ of actual. It became apparent in subsequent years, however, that the funnel percentages could change dramatically; this was both possible and problematic in UGC's case in particular, with rolling admissions and no predetermined cohort size. The trajectories were less prone to dramatic shifts but there were shifts nonetheless. Using the actual matriculants once they were known to project backwards (as in the diagnostic reports described earlier) showed that the accuracy on the total annual enrollments across all categories was within 1\% in the first 3 years that the approach was implemented, but was over 3\% in 2 of the remaining 4 of the 7 years the approach was used prior to the merger. This meant that the average trajectories in some categories had changed; that change leads to the second insight of thinking of forecast errors not primarily as problems with the models (although updating the coefficients may be in order), but rather as diagnostic opportunities to investigate reasons for changes in historical patterns and inform remedial and prescriptive decision-making. The diagnostics for individual student categories can often be quite helpful for prescriptive purposes even in years when the total enrollment projections are very accurate since pattern changes in different categories might be meaningful and yet cancel each other out in their enrollment effects. The trajectories (and the funnel model as well) were ideal for this purpose in that their construction was fundamentally descriptive rather than purely predictive.

\section{CONCLUSIONS AND POSSIBLE ENHANCEMENTS}

Trajectories combined with new matriculant projections to greatly enhance UGC's ability to accurately project enrollments. The approach was relatively simple (using basic tabulations, multiplications, and summations) and was easy to update using readily available data. Specific course enrollment projections, active student projections, and total course enrollment projections were all obtained using the same data and basic approach. Diagnostic reports identified changes in student behavior that informed prescriptive decision-making. Faculty planning was enhanced in both the short and long terms. 
In terms of future model enhancements that would fit nicely with the trajectory approach, I had begun to look at two possible adjustments. As described, the trajectories were computed by averaging across all students in the database. First, I had looked at time series (via weighted moving averages or exponential smoothing) adjustments to computing the trajectories. Second, I had looked at conditional trajectories (similar to the conditional course probabilities of Balachandran and Gerwin [1973]) for continuing students based on the number of courses already completed. Although neither of these possible enhancements led to any useful updates during the time of use, I believe they could have done so eventually as the overall body of data aged. The conditional trajectories in particular would have benefited from more data since they essentially created more student categories, hence fewer data per category. Institutions with larger amounts of data might find these enhancements immediately effective. Although this article focused on the trajectories, the projections of new matriculants could perhaps have been enhanced using regression and/or time series approaches with explanatory variables.

An overall conclusion is that many of the approaches in the literature could possibly be used to both enhance the trajectories' accuracy and to combine effectively with them. The best way to combine approaches and the potential benefits would likely depend very much on the application. This is true as well for the broader question of whether the trajectories or any other of the methodologies previously suggested in the literature would be a useful addition to any institution's approach. If the setting has significant similarities to UGC's setting, I believe the use of trajectories could be very helpful.

\section{REFERENCES}

Balachandran, K.R., \& Gerwin, D. (1973). Variablework models for predicting course enrollments. Operations Research, 21, 823-834.

Chen, C. (2008). An integrated enrollment forecast model. IR Applications, 15, 1-18.

Gandy, R., Crosby, L., Luna, A., Kasper, D., \& Kendrick, S. (2019). Enrollment projection using Markov Chains: Detecting leaky pipes and the bulge in the BOA. AlR Professional File, 147, 21-38.

Hopkins, D.P., \& Massy, W.F. (1981). Planning models for colleges and universities. Stanford University Press.

Kraft, C.R., \& Jarvis, J.P. (2005) An adaptive model for predicting course enrollment. Thesis, Mathematical Sciences, Clemson University, 1-10.

Lavilles, R.Q., \& Arcilla, M.J. (2012). Enrollment forecasting for school management system. International Journal of Modeling and Optimization, 2(5), 563-566.

Link, R., \& Whitford, M. (2018). Which way is the wind blowing: forecast enrollment with projection models. Presented at 2018 AIR Forum 2018, Orlando, FL. http://www.buffalo.edu/content/dam/www/provost/ files/oia/Presentations/AIR/AIR20180531_Forecast_ Enrollment-Link.pdf.

Maryland Higher Education Commission. (2016). Enrollment projections: Maryland public colleges and universities. Maryland Higher Education Commission. https://mhec.maryland.gov/publications/ Documents/Research/AnnualReports/20162025EnrollmentProjections.pdf. 
Nicholls, M. (2007). Assessing the progress and the underlying nature of the flows of doctoral and master degree candidates using absorbing Markov chain. Higher Education, 53, 769-790.

Ognjanovic, I., Gasevic, D., \& Dawson, S. (2016). Using institutional data to predict student course selections in higher education. Internet and Higher Education, 29, 49-62.

Rahim, R., Ibrahim, H., Kasim, M., \& Adnan, F. (2013). Projection model of postgraduate student flow. Applied Mathematics and Information Sciences, 7(21), 383-387.

Redlinger L. J., Etheredge, S., \& Wiorkowski, J. (2013). Using applications, admission data to forecast enrollments. Presented at May 2013 AIR Conference, Long Beach, CA. https://www.utdallas.edu/ospa/files/ ForecastingAIR2013-04-30.pdf.

Reiss, E. (2012). Best practices in enrollment modeling: Navigating methodology and processes. Presented at 2012 FACRAO Conference, St. Augustine, FL. https://docplayer.net/9731840Best-practices-in-enrollment-modeling-navigatingmethodology-and-processes.html.

Rylee, C., \& Trusheim, D. (2004). Enrollment projections and the budget process: A technique for smart planning. Presented at the SCUP-39 2004 Conference in Toronto, Canada.

Shapiro, J. K., \& Bray, C. (2011). Improving retention and enrollment forecasting in part-time programs. Continuing Higher Education Review, 75, 121-129. 